\title{
Pneumomediastinum in dermatomyositis: association with cutaneous vasculopathy
}

\author{
Hajime Kono, Shigeko Inokuma, Hisanori Nakayama, Mie Suzuki
}

\begin{abstract}
Objectives-To study the pathogenesis of pneumomediastinum in polymyositis/ dermatomyositis (PM/DM).

Patients and methods-The clinical records of 48 patients with PM/DM were reviewed, focusing mainly on the presence of pneumomediastinum and cutaneous vasculopathy, and the chest radiographic changes. A patient with pneumomediastinum with a characteristic change in his bronchus is described in detail. Case reports of pneumomediastinum in $\mathbf{P M / D M}$ in English publications are reviewed.

Results-Among the 48 patients with PM/DM, pneumomediastinum was observed as a complication in four patients with $D M$ and none of the patients with PM. Three of the four patients with pneumomediastinum, but only six of the 44 patients without this complication, had associated cutaneous vasculopathy. There was a significant association of pneumomediastinum with cutaneous vasculopathy $(p=0.02)$ and younger age $(p=0.04)$, but not with the prevalence of lung disease. A 30 year old man (patient 1) with DM, who had interstitial pneumonitis and skin ulceration due to vasculopathy, developed pneumomediastinum. Fibreoptic bronchoscopy showed white plaques on the bronchial mucosa, which were confirmed by microscopic examination as representing subepithelial necrosis. A literature review showed 13 cases of $D M$ but no patient with PM with pneumomediastinum.
\end{abstract}

Conclusions-In patient 1, bronchial necrosis due to vasculopathy was strongly suspected as being responsible for the pneumomediastinum. The results suggest that pneumomediastinum was associated not with interstitial pneumonitis but with the complication of vasculopathy appearing as skin lesions in DM.

(Ann Rheum Dis 2000;59:372-376)

Dermatomyositis (DM) is a generalised disorder characterised by myositis and typical cutaneous findings. The lung is commonly affected, usually by interstitial pneumonitis. ${ }^{1}$ Although spontaneous pneumomediastinum has been reported as a rare complication in DM, the pathogenesis has not so far been clarified, and it is believed that pneumomediastinum is attributable to interstitial pneumonitis. ${ }^{2}$ We recently encountered a patient with DM with the complications of skin ulceration and pneu- momediastinum, in whom fibreoptic bronchoscopy showed necrosis of the bronchial wall. We were prompted, on seeing this patient, to review our patients with polymyositis (PM) and DM who had developed pneumomediastinum, and determine its association with cutaneous vasculopathy.

We also reviewed case reports of pneumomediastinum in PM/DM, to determine whether it was interstitial pneumonitis or vasculopathy including skin that was responsible for pneumomediastinum.

\section{Patients and methods}

PATIENTS

The clinical records of all patients admitted to the Tokyo Metropolitan Komagome Hospital from January 1986 to December 1998 with a diagnosis of PM/DM were reviewed. Forty eight cases fulfilled the criteria of Bohan and Peter $^{3}$ for the diagnosis of PM/DM. The primary parameters considered included age, sex, skin manifestations, laboratory abnormalities of serum creatine kinase $(\mathrm{CK})$ and aldolase (ALD) levels, electromyographic findings, muscle biopsy results, chest radiographic changes including pneumomediastinum, association with malignancy, treatment, and outcome. Cutaneous vasculopathy was defined as the presence of periungual infarction, pitting scar, or erosive or ulcerative lesions. Skin manifestations consisting only of the classical heliotrope rash or Gottron's sign were not included in the definition of cutaneous vasculopathy.

A patient with DM and pneumomediastinum and bronchial necrosis is described in detail.

\section{STATISTICAL ANALYSIS}

We used the conventional $\chi^{2}$ and Fisher's exact tests to analyse the qualitative differences, Student's $t$ test for the comparison of means in large samples of similar variance, and the nonparametric Mann-Whitney $U$ test for small samples. A value of $\mathrm{p}<0.05$ was considered as denoting significance.

\section{LITERATURE REVIEW}

Case reports of patients with $\mathrm{PM} / \mathrm{DM}$ who developed pneumomediastinum were reviewed in English publications. We focused especially on the age, sex, diagnosis (PM or DM), peak serum levels of CK, association with cutaneous vasculopathy and pulmonary diseases, findings on bronchoscopy, treatment, and outcome.
Accepted for publication 2 November 1999 
Table 1 Review of 13 previously published and four present patients with polymyositis/dermatomyositis (PM/DM) complicated with pneumomediastinum

\begin{tabular}{|c|c|c|c|c|c|c|c|c|c|c|c|c|}
\hline Author & Ref & Diagnosis & $\begin{array}{l}\text { Age } \\
(y)\end{array}$ & $\operatorname{Sex}$ & $\begin{array}{l}C K^{\star} \\
(I U / l)\end{array}$ & $\begin{array}{l}\text { CK upper } \\
\text { normal } \\
\text { limit }\end{array}$ & $\begin{array}{l}\text { Cutaneous } \\
\text { vasculopathy }\end{array}$ & $\begin{array}{l}\text { Interstitial } \\
\text { pneumonitis }\end{array}$ & Bronchoscopy & Corticosteroid & Immunodepressant & Outcome \\
\hline Jansen TL & 2 & $\mathrm{DM}$ & 34 & $\mathrm{~F}$ & Normal & NA & No & Yes & NA & $\mathrm{PSL}^{\star} 60 \mathrm{mg}$ daily & MTX $15 \mathrm{mg} / \mathrm{w}$ & Alive \\
\hline Santiago MB & 4 & $\mathrm{DM}$ & 10 & M & Normal & NA & No & Yes & NA & $\begin{array}{l}\text { PSL } 1 \mathrm{mg} / \mathrm{kg}+ \\
\text { pulse }^{\star}\end{array}$ & $\begin{array}{l}\text { Hydroxychloroquine } \\
200 \mathrm{mg}\end{array}$ & Died \\
\hline Nagai Y & 5 & $\mathrm{DM}$ & 65 & $\mathrm{~F}$ & 2871 & 170 & No & Yes & NA & $\begin{array}{l}\text { Pulse+PSL } 60 \mathrm{mg} \\
\text { daily }\end{array}$ & No & Died \\
\hline Bousquet $\mathrm{E}$ & 6 & $\mathrm{DM}$ & 39 & M & Normal & NA & No & Yes & Normal & Pulse+PSL $1 \mathrm{mg} / \mathrm{kg}$ & $\mathrm{MTX}^{\star} 50 \mathrm{mg} / \mathrm{w}$ & Alive \\
\hline de Toro Santos FJ & 7 & $\mathrm{DM}$ & 41 & $\mathrm{~F}$ & Normal & NA & No & Yes & Normal & PSL $60 \mathrm{mg}$ daily & AZP $100 \mathrm{mg}$ & Died \\
\hline Isfer RS & 8 & $\mathrm{DM}$ & 25 & M & Normal & NA & Yes & No & Normal & PSL $50 \mathrm{mg}$ daily & Chloroquine $250 \mathrm{mg}$ & Unknown \\
\hline Matsuda Y & 9 & $\mathrm{DM}$ & 41 & $\mathrm{~F}$ & Normal & NA & No & Yes & NA & PSL $50 \mathrm{mg}$ daily & No & Died \\
\hline Cicuttini FM & 10 & $\mathrm{DM}$ & 22 & $\mathrm{~F}$ & Normal & NA & Yes & No & Normal & PSL $12.5 \mathrm{mg}$ daily & $\mathrm{AZP}^{\star} 75 \mathrm{mg}$ & Alive \\
\hline Carmody E & 11 & $\mathrm{DM}$ & 42 & M & 635 & 186 & Yes & No & Normal & PSL $60 \mathrm{mg}$ daily & $\mathrm{AZP}$ & Alive \\
\hline Bradley JD & 12 & $\mathrm{DM}$ & 20 & M & 2260 & 180 & Yes & Yes & NA & PSL $100 \mathrm{mg}$ daily & AZP $100 \mathrm{mg}$ & Alive \\
\hline Jang KA & 13 & $\mathrm{DM}$ & 32 & $\mathrm{~F}$ & NA & NA & Yes & Yes & NA & PSL $60 \mathrm{mg}$ daily & MTX $5 \mathrm{mg} / \mathrm{w}$ & Alive \\
\hline Yamanishi Y & 14 & $\mathrm{DM}$ & 23 & M & 170 & 120 & No & Yes & Normal & $\begin{array}{l}\text { PSL } 50 \mathrm{mg} \text { daily + } \\
\text { pulse }\end{array}$ & No & Died \\
\hline Yamanishi Y & 14 & $\mathrm{DM}$ & 57 & M & Normal & 120 & No & Yes & NA & $\begin{array}{l}\text { PSL } 50 \text { mg daily + } \\
\text { pulse }\end{array}$ & No & Died \\
\hline Patient 1 & & $\mathrm{DM}$ & 30 & M & 403 & 180 & Yes & Yes & Necrosis & PSL $15 \mathrm{mg}$ daily & $\mathrm{CSA}^{\star} 225 \mathrm{mg}$ & Alive \\
\hline Patient 2 & & $\mathrm{DM}$ & 25 & M & 377 & 180 & Yes & Yes & Normal & $\begin{array}{l}\text { PSL } 60 \mathrm{mg} \text { daily + } \\
\text { pulse }\end{array}$ & $\begin{array}{l}\text { AZP } 100 \mathrm{mg}, \text { CSA } \\
200 \mathrm{mg}\end{array}$ & Died \\
\hline Patient 3 & & $\mathrm{DM}$ & 23 & M & 219 & 180 & Yes & Yes & Not done & $\begin{array}{l}\text { PSL } 60 \text { mg daily + } \\
\text { pulse }\end{array}$ & No & Alive \\
\hline Patient 4 & & $\mathrm{DM}$ & 59 & $\mathrm{~F}$ & 3501 & 120 & No & Yes & $\begin{array}{l}\text { Bronchial stenosis } \\
\text { caused by old } \\
\text { tuberculosis }\end{array}$ & PSL 40 mg daily & No & Alive \\
\hline
\end{tabular}

${ }^{\star} \mathrm{CK}=$ creatine kinase PSL $=$ prednisolone; pulse = high dose steroid pulse treatment; $\mathrm{MTX}=$ methotrexate; $\mathrm{AZP}=$ azathioprine; $\mathrm{CSA}=$ cyclosporin $\mathrm{A} ; \mathrm{NA}=$ not available.

\section{Results}

RESULTS FOR CONSECUTIVE PATIENTS WITH PM/DM Forty eight patients with a diagnosis of PM/DM were followed up for a median duration of 37.5 months. The patients comprised 13 men and 35 women with a mean age at the time of diagnosis of 49 (SD 16, range 7-81) years. Of the 13 men, five had PM and eight had DM; of the 35 women, 15 had PM and 20 had DM. Ten patients, four with PM and six with DM, had an associated malignancy. In three patients with PM, myositis developed during the course of systemic sclerosis. Twenty eight (58\%) were still being followed up at the time of this review, 11 $(23 \%)$ had died, and nine $(19 \%)$ were lost to follow up. The causes of death included malignancy (five patients), interstitial pneumonitis (three), infection (one), scleroderma renal crisis (one), and unknown (one). Seven patients with DM and two patients with PM had cutaneous vasculopathy: six patients with periungual infarction, three with ulcerative or erosive lesions, and one with pitting scar. There was no statistical difference in the incidence of cutaneous vasculopathy between patients with PM and DM. Chest radiography showed abnormal findings in nine patients with PM and 19 patients with DM. Two patients with DM showed emphysematous change, two with DM had infiltrative opacities caused by aspiration pneu-

Table 2 Comparison of patients with and without pneumomediastinum in polymyositis/dermatomyositis (PM/DM)

\begin{tabular}{|c|c|c|c|}
\hline & $\begin{array}{l}\text { With pneumomediastinum } \\
\text { (patients } 1,2,3,4)\end{array}$ & $\begin{array}{l}\text { Without } \\
\text { pneumomediastinum }\end{array}$ & $p$ Value \\
\hline Number & 4 & 44 & \\
\hline Male/female & $3 / 1$ & $10 / 34$ & NS \\
\hline $\mathrm{Age}^{\star}$ & $34(17)$ & $51(15)$ & $<0.05$ \\
\hline Diagnosis (PM/DM) & $0 / 4$ & $20 / 24$ & NS \\
\hline Cutaneous vasculopathy & 3 & 6 & $<0.05$ \\
\hline Lung disease & 4 & 24 & NS \\
\hline Creatine kinase $(\mathrm{IU} / \mathrm{l})^{\star}$ & $1125(1186)$ & $3142(5661)$ & NS \\
\hline Aldolase $(\mathrm{IU} / \mathrm{l})^{\star}$ & $9.7(3.2)$ & $16.7(32.6)$ & NS \\
\hline Outcome (alive/died/lost to follow up) & $3 / 1 / 0$ & $25 / 10 / 9$ & NS \\
\hline
\end{tabular}

^Data shown as mean (SD). monia, and the remainder had findings of interstitial pneumonitis. The mean peak level of CK was $2974 \mathrm{IU} / 1$ (SD 5459) for all patients, 4126 IU/1 (SD 5991) for patients with PM, and 2151 IU/1 (SD 4992) for patients with DM. The mean peak level of ALD was $15.8 \mathrm{IU} / 1$ (SD 30.6) for all patients, 17.3 IU/1 (SD 24.1) for patients with PM, 15.3 IU/1 (SD 35.6) for patients with DM. There were no statistically significant differences in the levels of $\mathrm{CK}$ and ALD between patients with PM and DM.

Pneumomediastinum occurred in four patients with DM and none of the patients with PM (table 1). Patient 1 is described in detail below. All four patients had interstitial pneumonitis, and one patient (patient 2) died of it. One of the patients (patient 4) had aspiration pneumonia due to pharyngeal dysphagia, confirmed by a barium swallow, and the other three had no findings suggestive of any infection. The pneumomediastinum occurred during the course of steroid treatment in all the patients. Three of the four patients (patients 1-3) had associated cutaneous vasculopathy. In patient 2 skin biopsy from the dorsum of the left metacarpophalangeal joint showed perivascular infiltration of lymphocytes in the upper dermis, and biopsy of the right rectus muscle showed disorganisation of the intermyofibrillar network on nicotinamide adenine dinucleotide-tetrazolium reductase staining. In patient 3 extremely deep ulcerations appeared on the ears and on the extensor surfaces of the metacarpophalangeal joints and elbows exposing the underlying tendons. These three patients were unique in that they were all young men (30, 25, 23 years) and had relatively low peak levels of CK $(403,377,219$ IU/1).

Table 2 shows the clinical features of the four patients with pneumomediastinum and of the remaining 44 patients without this complication. Three of the four patients with pneumomediastinum, but only six of the 44 patients 


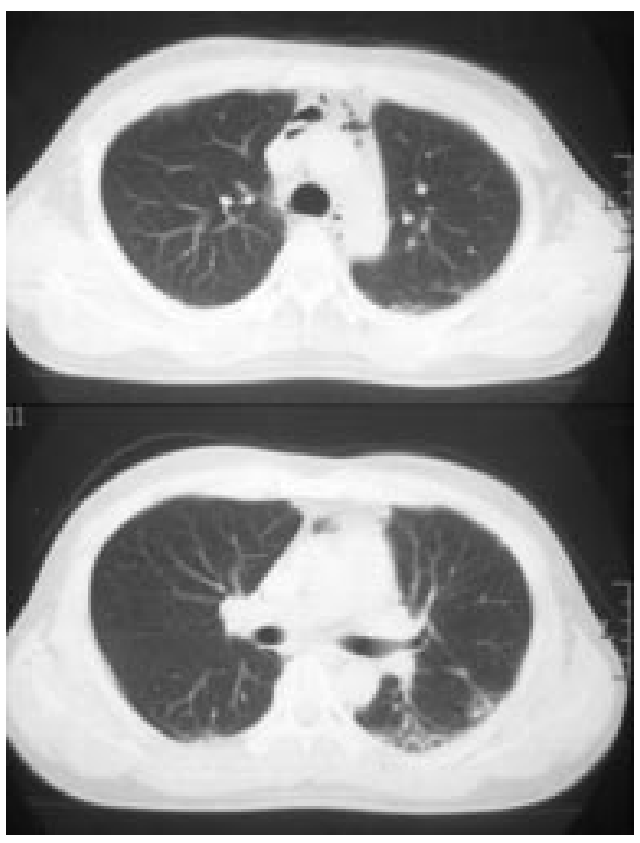

Figure 1 Computed tomograms of the chest (patient 1), showing air around the trachea and in the anterior mediastinum. A honeycomb pattern is also seen in the posterior bases of the lungs.

without it, had cutaneous vasculopathy. A significant association was found between the development of pneumomediastinum and the presence of cutaneous vasculopathy $(\mathrm{p}=0.02$, $\chi^{2}=5.5$ ). Patients with pneumomediastinum were significantly younger than those without it $(p=0.04)$. There were no differences between the two groups based on sex, the diagnosis (PM or DM), the prevalence of lung disease, outcome, association with a malignancy, or the peak serum levels of CK or ALD.

CASE REPORT

Patient 1, a 30 year old man, was admitted to another hospital in May 1998 with a two month history of muscle weakness, arthralgia, and fever of $38^{\circ} \mathrm{C}$. He had lost $12 \mathrm{~kg}$ in two months. DM was diagnosed based on the findings of typical Gottron's papules, proximal muscle weakness, raised serum levels of CK, myopathic pattern on electromyography, and the finding of interstitial pneumonitis. Biopsy of the left deltoid muscle showed no abnormal findings. Investigations for possible malignancy were negative. He was treated with prednisolone $50 \mathrm{mg}$ daily initially, followed by gradual tapering of the dose. The Gottron's papules faded and the serum levels of CK decreased, but interstitial pneumonitis continued to progress slowly. Pneumomediastinum and subcutaneous emphysema developed in July 1998 following a bout of cough, when he was receiving prednisolone at a dose of $15 \mathrm{mg}$ daily (fig 1), but subsided in a few days without any additional treatment. He was discharged from the hospital in July 1998.

He was referred to and admitted to our hospital in October 1998 with a two month history of recurrent symptoms of fever, cough, muscle weakness, malaise, and skin rash. On admission, physical examination showed a fever of $37.5^{\circ} \mathrm{C}$, heliotrope lesions on the upper eyelids and bridge of the nose, periungual erythema, and an erythematous, scaling rash on the dorsum of both hands, elbows, shoulders, and back. The erythematous lesions on the back were ulcerated. Oral ulcers, angular stomatitis, and haemoptysis were noted after admission. His proximal muscles in both legs and arms were determined to have a power of grade $4 / 5$. There were fine inspiratory rales in both lower lung regions, and no wheeze.

Urine analysis was normal. Haematological examination showed a white cell count of $5.3 \times$ $10 \%$ (81.6\% segmented neutrophils, $0.2 \%$ eosinophils, $0.6 \%$ basophils, $6.9 \%$ monocytes, and $10.7 \%$ lymphocytes), a haemoglobin level of $146 \mathrm{~g} / 1$, and a platelet count of $181 \times 10^{9} / 1$. Tests for antinuclear antibodies, anti-double stranded DNA and anti-Jo-1 were negative. Raised levels of serum CK of 403 IU/1 (normal value $<180$ ), ALD of $7.6 \mathrm{IU} / 1$ (normal value $<3.1$ ), aspartate aminotransferase of $50 \mathrm{IU} / 1$ (AST; normal value $<32$ ), alanine aminotransferase of $80 \mathrm{IU} / 1$ (ALT; normal value <43), and lactate dehydrogenase of 311 IU/1 (LDH; normal value $<220$ ) were found. A room-air blood gas showed a $\mathrm{PO}_{2}$ of $78 \mathrm{~mm} \mathrm{Hg}$ and a $\mathrm{Paco}_{2}$ of $34 \mathrm{~mm} \mathrm{Hg}$.

A plain roentgenogram and computed tomograms of the chest showed increased interstitial markings in the lower lung fields of both lungs. Pulmonary function tests disclosed a decreased vital capacity of 2.82 litre $(70.1 \%$ of predicted value), and a steady state diffusing capacity of $10.7 \mathrm{ml} / \mathrm{min} / \mathrm{mm} \mathrm{Hg}(63.0 \%$ of the predicted value). Fibreoptic bronchoscopy showed studded white plaques on the bronchial mucosa at the carina and the main, lobar, and segmental bronchi in both lungs (figs $2 \mathrm{~A}$ and B). Biopsy of the plaque at the carina showed subepithelial necrosis of the bronchial wall with epithelial squamatisation (fig 3). Neither bacteria nor fungi were found by Grocott and Gram staining. No vasculature was included in the bronchial biopsy specimen. Bronchoalveolar lavage cell counts showed $88 \%$ macrophages, $7 \%$ lymphocytes, and 5\% neutrophils. Biopsy of an erythematous skin lesion with ulceration on the back showed perivascular infiltration of lymphocytes and foamy histiocytes in the upper dermis, with negative immunofluorescence for immunoglobulins $\mathrm{G}, \mathrm{M}$, and $\mathrm{A}$.

$\mathrm{He}$ was treated with cyclosporin $225 \mathrm{mg}$ daily, in addition to successive doses of prednisolone $15 \mathrm{mg}$ daily. The levels of CK, ALD, AST, ALT, and LDH returned to normal, and muscle weakness and ulceration of the skin also gradually improved. Haemoptysis stopped and stomatitis disappeared, and he gained weight. However, the interstitial markings on the chest roentgenograms and the decreased vital capacity and diffusing capacity in pulmonary function tests did not change. Repeat bronchoscopy was not performed.

LITERATURE REVIEW

Thirteen case reports of spontaneous pneumomediastinum in patients with $\mathrm{PM} / \mathrm{DM}$ were identified in the English literature, ${ }^{24-14}$ in which sufficient clinical information was provided to 

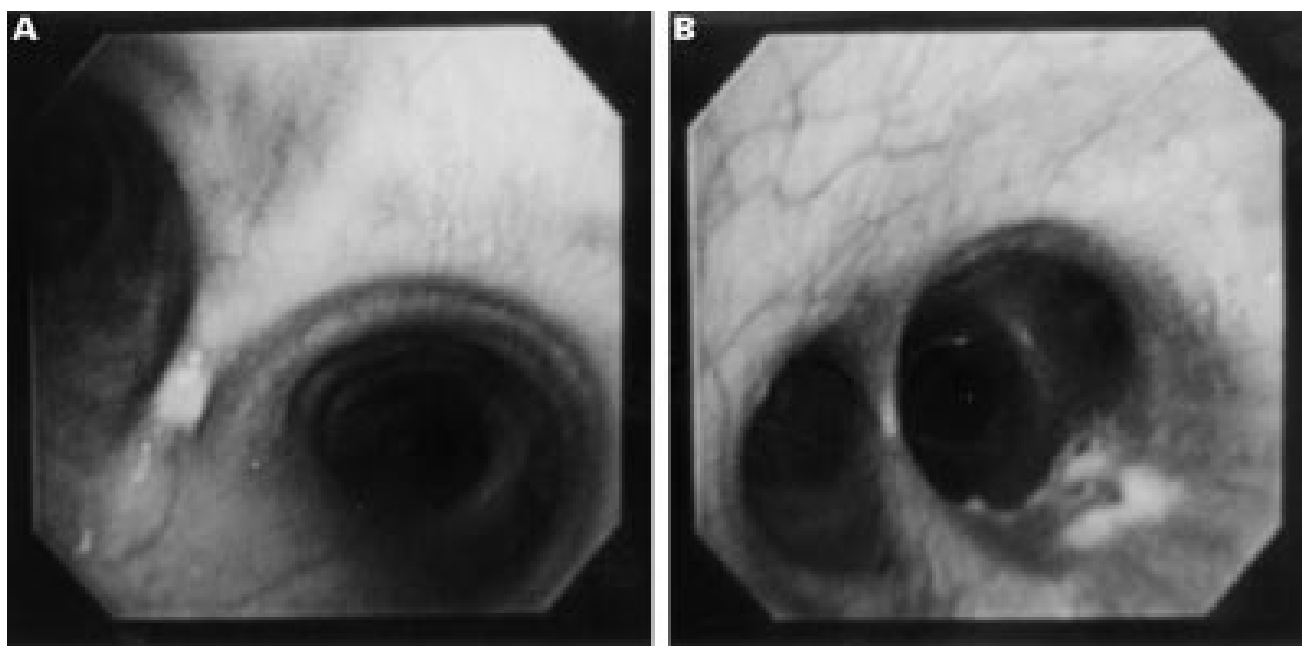

Figure 2 A fibreoptic bronchoscopy, showing studded white plaques on the bronchial mucosa at the carina $(A)$ and the main (B), lobar and segmental bronchi of both lungs.

allow their inclusion in table 1 . Table 1 also includes the four patients whom we encountered. The diagnosis in all these 17 patients was $\mathrm{DM}$, and no case report of PM with pneumomediastinum has been reported. The serum levels of CK were normal in eight patients. Cutaneous vasculopathy was reported in eight patients. Fourteen patients had interstitial pneumonitis and three did not. Fibreoptic bronchoscopy showed no abnormal findings other than in patient 1 , as described above. All patients were treated by conventional steroid treatment with or without high dose steroid pulses or other immunosuppressants. Except for one patient, ${ }^{8}$ pneumomediastinum occurred during steroid treatment. Seven patients died; 6 patients of respiratory failure and one patient of infection.

\section{Discussion}

PM/DM are commonly associated with pulmonary disease, including the usual interstitial pneumonitis, diffuse alveolar damage, and bronchiolitis obliterans with organising pneumonia. ${ }^{15}$ Although the exact prevalence is not known, it has been reported that $39 \%$ of

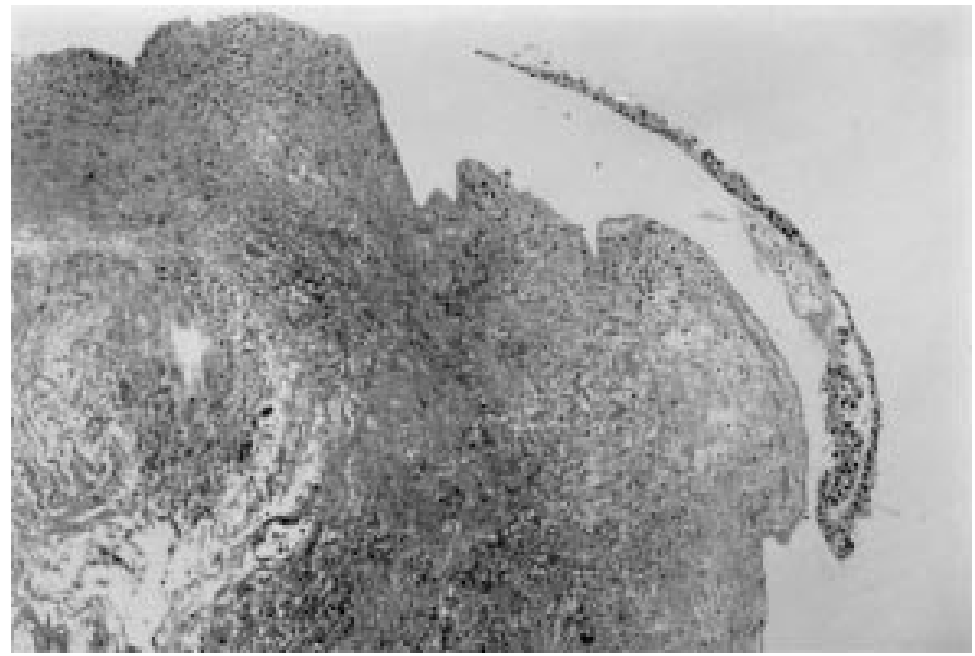

Figure 3 Biopsy of the plaque at the carina (original magnification $\times 60$, haematoxylin and eosin staining) showing subepithelial necrosis of the bronchial wall with epithelial squamatisation. patients with $\mathrm{PM} / \mathrm{DM}$ have interstitial lung disease (ILD). ${ }^{1}$ No reported case of PM/DM with necrotic lesions of the bronchial wall was found. Pneumomediastinum is a rare complication in $\mathrm{PM} / \mathrm{DM},{ }^{16}$ but the literature review confirmed 13 cases of DM with this complication. There is, to date, no report of PM with pneumomediastinum. Jansen et al reported that pneumomediastinum appeared to occur primarily in patients with pre-existing lung disease. ${ }^{2}$ ILD could be complicated by pneumomediastinum; McLoud et al reported an incidence of $7.4 \%$ among patients with usual interstitial pneumonitis. ${ }^{17}$ Although all of our four patients had ILD, three ${ }^{810}$ of the 13 cases with pneumomediastinum reported in the literature had no finding of ILD, suggesting that it is not an essential underlying condition for the development of pneumomediastinum. However, because of the high incidence (14 out 17) of ILD among patients with pneumomediastinum, it is difficult to exclude ILD as a possible contributory cause.

In general, three pathological situations have been considered to give rise potentially to pneumomediastinum: (a) soft tissue infections by gas forming microorganisms; (b) disruption of cutaneous or mucosal barriers; and (c) rupture of the alveoli due to a marked increase in intra-alveolar pressure or a decrease in peribronchovascular interstitial pressure, or both. ${ }^{18}$ Five reported cases (the three cases without ILD $^{81011}$ and two cases with ILD $^{1213}$ ) had ulcerative or vasculitic lesions of the skin, indicating the existence of associated ischaemic vasculopathy. Our three patients with pneumomediastinum also had cutaneous vasculopathy. The prevalence of the vasculopathy was significantly higher in patients with pneumomediastinum than in those without it. This suggests that vasculopathy may be an important factor leading to disruption of the bronchial mucosal barrier and causing development of pneumomediastinum in DM.

The presented literature review and the findings in our patients show that pneumomediastinum characteristically occurs only in patients with DM and never in those with PM. 
The findings in the muscle biopsy specimens from patients with $\mathrm{PM} / \mathrm{DM}$ indicate a tendency for different classes of lymphocytes to accumulate in different regions of the muscle. ${ }^{19}$ In DM, B cells and CD4+ $\mathrm{T}$ cells were abundant in the perivascular regions, with deposition of late complement components and capillary loss. ${ }^{20}$ The vascular pathology and the more prominent perifascicular atrophy in DM suggested that the primary pathology was a vasculopathy. ${ }^{16}$ In our series and in the studies reviewed, almost all cases but one developed pneumomediastinum during steroid treatment, suggesting that steroids also have a causative role.

That the prevalence of ILD was similar in $\mathrm{PM}$ and in DM in our series, and in the literature as well, ${ }^{1}$ is not consistent with the assumption that pneumomediastinum is caused by ILD. The absence of any statistically significant difference in the prevalence of pneumomediastinum or cutaneous vasculopathy between PM and DM in our study is probably due to the small number of patients studied.

No case of DM with necrotic lesions of the bronchial wall has been reported in the English literature. Necrosis of the bronchial wall has been reported in cases with radiation treatment, ${ }^{21}$ chronic $\mathrm{Q}$ fever ${ }^{22}$ prolonged intake of Sauropus androgynus, ${ }^{23}$ necrotising tracheobronchitis in neonates, ${ }^{24}$ ingestion of caustic substances, ${ }^{25}$ and Wegener's granulomatosis. ${ }^{26}$ The possible causes of the bronchial necrosis in these cases were classified as direct invasion or damage, or vascular impairment. In patient 1 , as the ulceration of the skin was caused by ischaemic vasculopathy, the bronchial wall necrosis might also be considered as being caused by focal ischaemia with vasculopathy. Necrosis of the bronchial wall could cause an air leak resulting in pneumomediastinum.

In summary, we described a patient with DM who had ulcerative skin lesions attributable to vasculopathy, bronchial necrosis, and ILD, who developed the complication of pneumomediastinum. It is assumed that the necrosis of the bronchial wall due to vasculopathy could be the cause of his pneumomediastinum. This report offers, for the first time, a plausible mechanism for the pathogenesis of pneumomediastinum in DM. We also indicate that pneumomediastinum is associated not with interstitial pneumonitis but with cutaneous vasculopathy. It is suggested that pneumomediastinum occurs only in cases of DM associated with vasculopathy, which could easily be observed as a skin manifestation.

1 Lakhanpal S, Lie JT, Conn DL, Martin WJ 2d. Pulmonary disease in polymyositis/dermatomyositis: a clinicopathological analysis of 65 autopsy cases. Ann Rheum Dis 1987;46:23-9.
2 Jansen TL, Barrera P, van Engelen BG, Cox N, Laan RF, van de Putte LB. Dermatomyositis with subclinical myositis and spontaneous pneumomediastinum with pneumothorax: case report and review of the literature. Clin Exp Rheumatol 1998;16:733-5.

3 Bohan A, Peter JB. Polymyositis and dermatomyositis (parts 1 and 2). N Engl J Med 1975;292:344-7, 403-7.

4 Santiago MB, Chalhoub M, Pereira ST. Amyopathic dermatomyositis complicated by interstitial pulmonary disease and pneumomediastinum. J Rheumatol 1998;25: 2042-3

5 Nagai Y, Ishikawa O, Miyachi Y. Pneumomediastinum and subcutaneous emphysema associated with fatal interstitial pneumonia in dermatomyositis. J Dermatol 1997;24: $482-4$.

6 Bousquet E, Rolland Y, Bon E, Cantagrel A, Tubery M, Murris M, et al. Pneumomediastinum, a rare complication of dermatomyositis. Report of a case. Rev Rhum Engl Ed 1996;63:457-9.

7 de Toro Santos FJ, Verea-Hernando H, Montero C, BlancoAparicio M, Torres Lanzas J, Pombo Felipe F. Chronic pneumomediastinum and subcutaneous emphysema: association with dermatomyositis. Respiration 1995;62:53-6.

8 Isfer RS, Nakao SK, Cernea SS. Dermatomyositis associated with pneumomediastinum and subcutaneous cellular tissue emphysema. Br J Rheumatol 1994;33:1092.

9 Matsuda Y, Tomii M, Kashiwazaki S. Fatal pneumomediastinum in dermatomyositis without creatine kinase elevation. Intern Med 1993;32:643-7.

10 Cicuttini FM, Fraser KJ. Recurrent pneumomediastinum in adult dermatomyositis. J Rheumatol 1989;16:384-6.

11 Carmody E, McNicholl J, Chadwick G, Bresnihan B, Fitzgerald MX. Prolonged spontaneous pneumomediastinum in adult dermatomyositis. Ann Rheum Dis 1987;46: 566.

12 Bradley JD. Spontaneous pneumomediastinum in adult dermatomyositis. Ann Rheum Dis 1986;45:780-2.

13 Jang KA, Kim SH, Choi JH, Sung KJ, Moon KC, Koh JK. Subcutaneous emphysema with spontaneous pneumomediastinum and pneumothorax in adult dermatomyositis. J Dermatol 1999;26:125-7.

14 Yamanishi Y, Maeda H, Konishi F, Hiyama K, Yamana S, Ishioka $S$, et al. Dermatomyositis associated with rapidly progressive fatal interstitial pneumonitis and pneumomediastinum. Scand J Rheumatol 1999;28:58-61.

15 Tazelaar HD, Viggiano RW, Pickersgill J, Colby TV. Interstitial lung disease in polymyositis and dermatomyositis. Am Rev Respir Dis 1990;141:727-33.

16 Miller FW. Inflammatory myopathies: polymyositis, dermatomyositis, and related conditions. In: Koopman WJ, ed. Arthritis and allied conditions. Maryland: Williams and Wilkins, 1997:1407-31.

17 McLoud TC, Carrington CB, Gaensler EA. Diffuse infiltrative lung disease: a new scheme for description. Radiology 1983;149:353-63.

18 Maunder RJ, Pierson DJ, Hudson LD. Subcutaneous and mediastinal emphysema. Pathophysiology, diagnosis, and management. Arch Intern Med 1984;144:1447-53.

19 Engel AG, Arahata K. Mononuclear cells in myopathies: quantitation of functionally distinct subsets, recognition of antigen-specific cell-mediated cytotoxicity in some disease, and implications for the pathogenesis of the different inflammatory myopathies. Hum Pathol 1986;17:704-21.

20 Kissel JT, Mendell JR, Rammohan KW. Microvascular deposition of complement membrane attack complex in dermatomyositis. N Engl J Med 1986;314:329-34.

21 Mehta AC, Dweik RA. Necrosis of the bronchus: role of radiation. Chest 1995;108:1462-6.

22 Kayser K, Wiebel M, Schulz V, Gabius HJ. Necrotizing bronchitis, angiitis, and amyloidosis associated with chronic Q fever. Respiration 1995;62:114-16.

23 Chang YL, Yao YT, Wang NS, Lee YC. Segmental necrosis of small bronchi after prolonged intakes of Sauropus androgynus in Taiwan. Am J Respir Crit Care Med 1998;157:594-8.

24 Hanson JB, Waldstein G, Hernandez JA, Fan LL. Necrotizing tracheobronchitis: an ischemic lesion. Am J Dis Child 1988;142:1094-8.

25 Lamaze R, Tréchot P, Martinet Y. Bronchial necrosis and granuloma induced by aspiration of a tablet of ferrous sulphate. Eur Respir J 1994;7:1710-11.

26 Travis WD, Hoffman GS, Leavitt RY, Pass HI, Fauci AS. Surgical pathology of the lung in Wegener's granulomatosis. Review of 87 open lung biopsies from 67 patients. Am J Surg Pathol 1991;15:315-33. 